Thélème. Revista Complutense de Estudios Franceses

ISSN-e: $1989-8193$

http://dx.doi.org/10.5209/THEL.55737

\title{
La réécriture des parémies : perspectives phraséologique et phraséodidactique
}

\author{
Riham El Khamissy ${ }^{1}$
}

Recibido: 09 de abril de 2017 / Aceptado: 08 de mayo 2017

Résumé. Notre article est axé sur l'étude des parémies qui sont la cible-vedette de réécriture dans le discours journalistique. Nous posons, dans un premier temps, la question de la compréhension : la stabilité/instabilité du sens d'un énoncé parémique due à la variabilité des formes qui le composent et de son contexte d'emploi. Une question linguistique (connaître les procédés de variations et de détournements) qui, surtout lorsqu'on l'étudie dans un corpus médiatique, devient également une problématique culturelle, pragmatique et communicative qui exige une lecture attentive. Ensuite, la réécriture des parémies est examinée du point de vue de la phraséodidactique pour les locuteurs non-confirmés. Elle concerne les compétences de compréhension et de production des écrits dans l'acquisition / apprentissage de la langue seconde ou étrangère.

Mots clés : phraséologie; proverbes; pragmatique; presse; didactique.

\section{[es] Paremia reescritura: fraseológico y fraseodidáctica perspectiva}

Resumen. Nuestro trabajo se centra en el estudio de paremias que son el objetivo-estrella de volver a escribir en el discurso periodístico. Nos preguntamos, en primera instancia, la cuestión de la comprensión: la estabilidad / inestabilidad del significado de una paremia debido a la variabilidad de las formas que componen el trabajo y su contexto. Una cuestión de lengua (sobre los procesos de cambio y desvíos) que, en el discurso periodístico, se convierte en una cuestión cultural, pragmática y comunicativa que requiere una lectura cuidadosa. A continuación, volver a escribir paremias se examina desde la perspectiva de fraseología y de didáctica para hablantes no nativos. Se refiere a las habilidades de comprensión y producción escrita en la adquisición / aprendizaje de una segunda lengua o lengua extranjera.

Palabras clave: fraseología; proverbios; pragmáticamente; periodístico-didáctica.

\section{[en] The Rewriting of Idioms: Phraseological and Didactic Perspective}

\begin{abstract}
This paper focuses on proverbs and sayings that are subject to re-writing in journalistic language. Studying this phenomenon in the French journalistic discourse is a linguistic issue which examines the stability/instability of a given proverb's meaning. Such variations might be due to the shifting of the proverb's elements and forms. When applied to a media corpus this phenomenon also becomes a cultural, pragmatic and cultural matter that demands careful scrutiny. In keeping with such an objective, the rewriting of proverbs is analyzed from a didactic perspective for non-native speakers. The analysis is concerned with comprehension, expression, and syntactic skills in the second/foreign language acquisition/learning.
\end{abstract}

Keywords: phraseology; proverbs; pragmatic; press-didactics.

Université Ain Shams, Le Caire, Égypte

rihamelkhamissy@yahoo.fr 
Sommaire : 1. Introduction. 2. Problème de taxinomie. 3. Incomplétude des parémies dans la presse. 4. Détournement des parémies dans la presse. 4.1. Détournement par substitution. 4.1.1. Substitution fondée sur le signifiant. 4.1.2. Substitution lexicale. 4.1.3. Substitution syntaxique. 4.2. Détournement non marqué : l'expansion. 5. Phraséodidactique du dé-(figement). 5.1. Construction du savoir. 5.2. Phase de production. 6. Conclusion, 7. Références bibliographiques.

Cómo citar: El Khamissy, R. (2017). « La réécriture des parémies : perspectives phraséologique et phraséodidactique ». Thélème. Revista Complutense de Estudios Franceses, Vol. 32, Núm. 2 : 175-196.

\section{Introduction}

La parémiologie constitue un domaine " vaste » et « éclaté » (Conenna, $1988: 99)$ lié à des disciplines linguistiques, sociales et didactiques. La presse écrite procure une vaste panoplie d'occurrences de parémies et représente le lieu privilégié d'actualisation des procédés de réécriture. Nous posons, dans la présente recherche, la question de la compréhension : la stabilité/instabilité du sens d'un énoncé parémique due à la variabilité des formes qui le composent et de son contexte d'emploi. Une question linguistique (connaître les procédés de détournements) qui, surtout lorsqu'on l'étudie dans un corpus médiatique, devient également culturelle, pragmatique et communicative. On se demandera, par exemple, ce qui arriverait si tous les chemins ne menaient pas à Rome, mais plutôt à Schengen, quand est-ce que l'habit ne fait pas le moine, ni le républicain et comment sans le chat, les souris dépriment. Quel effet sémantico-pragmatique produira un homme averti en vaudou, ou encore au pays des aveugles, le borgne fait führer? La finalité de ce travail réside dans la mise en lumière des procédés de réécriture des proverbes dans la presse, leur décodage par le récepteur, l'effet produit sur ce dernier.

De la lecture interprétative, nous passerons à la didactique. Les séquences figées sont souvent reléguées à un apprentissage par cœur de leur forme et de leur sens. Elles sont abordées en tant qu'objet d'apprentissage, hors contexte et sans activités pédagogiques importantes. Nous proposons ici de défiger les parémies en vue d'obtenir de nouvelles formulations qui s'écartent plus ou moins de la parémie-mère.

Nous ne manquerons pas de souligner, via la présente étude, l'inventivité et la nouveauté qu'introduit la réécriture dans le domaine de la phraséodidactique.

\section{Problème de taxinomie}

Le problème de la définition des énoncés sentencieux et parémiques est un débat interminable. Nombreux sont les linguistes qui se sont penchés surtout sur la définition du proverbe. Le proverbe est « un fait de langue » et «si on se focalise sur le proverbe on relève que c'est au fil de ses nombreuses attestations, suivant les étapes de ses mutations que se définit sa propre identité » (Conenna, 2002:35). Une des définitions les plus récentes du proverbe, celle de Petitjean et Pétillon $(2013: 297)$, nous laisse perplexe par son vague et sa généralité : «Énoncé concis, utilisant un présent dit générique et formulant un principe général dans le but de rendre compte 
d'une situation particulière, cette phrase possède les attributs majeurs d'une parole proverbiale ». Certains linguistes, comme Mejri, précisent que « le proverbe idéal serait un énoncé qui répondrait aux trois structurations prosodique ${ }^{2}$, syntaxique ${ }^{3}$ et sémantique $^{4} \gg(2008$ : 175). Dans les travaux d'Anscombre (1989; 1994), approfondis par Gomez-Jordana (2012) et par Petitjean et Pétillon (2013), tout proverbe est un « proverbe-topos », stéréotype à valeur argumentative dont la structure sémantico-pragmatique équivaut à la formule / $\mathrm{P}$ (proverbe) est un argument pour une conclusion Q (souvent implicite) /. Or, il est des cas où les parémies ne se contentent pas de sous-tendre un seul schéma argumentatif, mais deux, voire plusieurs. Telle est la conception de Fournet-Pérot (2013 : 192) dont la recherche rédigée en français et explorant les proverbes espagnols démontre que certains proverbes mènent à « des conclusions factuelles (CF) distinctes parfaitement déconnectées les unes des autres) ».

Par ailleurs, le statut même du proverbe en tant qu' « énoncé » n'a pas l'unanimité des linguistes. Selon Kleiber (1989, 1999), le proverbe est un nom, plus précisément une dénomination métalinguistique au même titre qu'un nom commun, tandis que pour Anscombre (1994 : 104), le proverbe est une proposition (ou une phrase) dont l'une des fonctions est la dénomination. Christine Michaux, quant à elle, y voit à la fois un nom et une phrase : « le proverbe est un nom : par conséquent, son énonciation convoque une représentation mentale préconstruite. Mais à un niveau supérieur, le proverbe est aussi une phrase, et il se voit donc associer une forme logique qui permet l'accès au calcul compositionnel du sens de l'énoncé » (1999 : 343). Elle rejoint ainsi la deuxième position de Kleiber sur la question du proverbe (une dénomination et une phrase) : «La particularité fondamentale d'un proverbe est d'être (...) à la fois, une dénomination (relative fixité de la forme, apprentissage, présence dans les dictionnaires, (relative) opacité, etc.), c'est-à-dire une entité codée, faisant partie du code linguistique, en ce qu'elle nomme une entité générale et non un particulier, et une phrase » (Kleiber, $1999: 64)$.

En outre, la frontière n'est pas étanche chez tous les linguistes, entre le proverbe et des formes apparentées comme le dicton, la maxime etc. Rodegem et Brussel (1989 : 349) proposent une catégorisation assez intéressante des parémies parce qu'elle précise clairement les nuances qui distinguent chaque catégorie :

(...) nous appelons « parémies » $(\mathrm{P})$ les citations orales à caractère normatif et rien qu'elles. Les parémies se subdivisent en deux groupes distincts selon leur contenu : enseignement moral et recettes de vie pratique. La morale, au sens large, regroupe le proverbe $(\mathrm{P} 1)$, la locution proverbiale $(\mathrm{P} 2)$, la maxime $(\mathrm{P} 3)$ et l'aphorisme (P4). Les notions véhiculées par ces types d'énoncés sentencieux peuvent s'appliquer à tous les hommes, puisque chacun de ces micro-récits exprime un universel à propos de l'homme. Telle est leur caractéristique commune. Et la norme est dite générale. Lorsque les énoncés sentencieux s'appliquent à des groupes distincts d'individus, la norme est dite restreinte. Appartiennent à ce secteur : le dicton (P5), l'adage juridique (P6), le slogan (P7) et la devise (P8). Hors cadre,

Obéissant souvent à une fixité rythmique.

Une syntaxe qui privilégie la concision (absence de déterminants ou article zéro, emploi de la relative nominale, économie de l'élément verbal, emploi du présent de l'indicatif.

4 Énoncés valides dans tout univers de croyance. 
et par souci d'être exhaustive, nous avons retenu un genre marginal, comprenant l'apophtegme (P9), énoncé signé, et le Wellérisme ${ }^{5}$ (P10), énoncé parodique (Rodegem et Brussel, 1989 : 349).

Pour plus de minutie, Kleiber (2000 : 46) propose la distinction entre proverbes et dictons basée sur le trait sémantique $\{ \pm \text { humain }\}^{6}$, et Schapira (1997: 89) opte pour la distinction entre proverbes et maximes fondée sur le trait sémantique $\{ \pm$ anonyme .

Nous écarterons de nos analyses le slogan politique et publicitaire qui se rapprochent tous deux trop du proverbe dans sa construction rythmique et métaphorique : bien que le slogan soit une formule à effet, il se distancie du proverbe par le fait qu'il manque de pérennité et s'apparente plutôt à l'hapax puisqu'il n'a pas l'atout d'être ancré dans la mémoire collective ou le legs culturel populaire.

Toutes les parémies se caractérisent par une brièveté formelle, une densité sémantique, un certain degré de figement ${ }^{7}$, et correspondent au syntagme figé de Saussure (1974 : 158). Le figement des parémies est aisément détecté par tout locuteur natif : " Il existe, au même titre que le sentiment de grammaticalité, un sentiment de figement chez la plupart des locuteurs natifs d'une langue. (...) tout locuteur natif, instruit et formé à l'art de varier son expression et de l'adapter à différentes situations de communication, peut assez aisément identifier, à l'intérieur d'un discours, un ensemble de séquences qu'il jugera figées et les distinguer d'un autre ensemble qu'il jugera, sinon libres, tout au moins normales » (Ibrahim, 1998 : 374).

Selon Fiala (1987 : 32), les unités phraséologiques, entre autres les parémies, sont des « combinaisons récurrentes, plus ou moins stabilisées, de formes lexicales et grammaticales ». Ces unités polylexicales ou phrases figées « ne sont qu'exceptionnellement entièrement figées »(Gross, 1988 : 22).

Les parémies sont en principe comprises des locuteurs confirmés. Défigées, elles posent des énigmes moins faciles à élucider par les récepteurs, notamment les locuteurs non-confirmés.

Notre travail s'appuie sur un corpus composé de proverbes et dictons parmi les plus utilisés et qui, selon nos propres observations, sont la cible-vedette de réécriture et de modification ${ }^{8}$. Nous verrons comment les modifications que ces parémies ont subies laissent le destinataire inassouvi (cas d'inachèvement) ou éconduisent ses attentes (cas de détournement). Dans le premier cas, le locuteur s'abstient volontai-

«Le wellérisme présente une structure tripartite :

- une remarque (sous forme de citation d'un proverbe ou d'un pseudo-proverbe)

- une attribution (« dit/a dit/disait Untel» : personne, animal, rarement chose)

- une situation (cette troisième partie semble facultative ; néanmoins, elle est souvent nécessaire pour détourner le message de la première partie en le mettant en opposition avec la situation donnée) » (Barta, 2006 :66).

Cette hypothèse longuement défendue par Kleiber (2000) qui permettait d'établir une distinction entre proverbe et dicton en ce sens qu'un proverbe doit porter sur les humains se trouve remise en question par Kleiber (2009).

Équivalent d'inaltérabilité chez Bally (1905: 87).

8 Un test sur le moteur de recherche Google montre que, sur Internet, les parémies étudiées sont parmi les plus utilisées et les plus détournées. Pour effectuer ce test, il suffit de taper l'énoncé parémique entre guillemets. Certes, il faut prendre en compte, en examinant les résultats, une marge d'évolution dans les statistiques. Reste que ce test fournit au chercheur une idée sur la fréquence de l'usage de la parémie et des modifications qu'elle subit. 
rement de citer entièrement la parémie. Dans le second, le locuteur dit autre chose, effectue des modifications, faisant ainsi un clin d'œil aux destinataires.

\section{Incomplétude des parémies dans la presse}

Une parémie inachevée, au lieu de dire, suggère. Partant du principe de la concision éloquente qui se trouve à la base de la construction d'une grande partie des proverbes et dictons, l'inachèvement de ces parémies s'observe de plus en plus. Les structures elliptiques, dans les titres de presse, ont toutes pour point commun la disparition de la seconde moitié de la parémie. Se cache, pensons-nous, derrière le choix d'un hypotexte célèbre comme titre, une tendance à une lecture (re)-compositionnelle de la part du récepteur. Examinons la construction suivante :

\section{(1) «Quand le chat n'est pas là »}

Cet énoncé est le titre d'articles parus respectivement dans Le Figaro du 8/12/2001, Le progrès du 14/3/2007, Revue de l'automobile du 19/10/2006, L'Équipe du 8/6/2007, Le Matin du 27/9/2008. Il s'agit de souligner l'absence, tant par le sens ( «le chat n'est pas là ») que par la forme (absence de la suite du proverbe), et de sous-entendre une conséquence à cet absentéisme. L'invitation à la reconstruction du modèle proverbial, dans sa forme canonique et complète, est bien présente (Quand le chat n'est pas là, les souris dansent). Il y a ici stabilisation d'un sémantisme, celui de l'absence. Toutefois, une panoplie de possibilités pour les conséquences les plus inattendues est offerte. L'identification des référents du chat qui n'est pas là est assez aisée en contexte linguistique : dans l'article du Figaro, c'est l'absence des deux équipes favorites du Quinté, à savoir Varenne qui est au départ du Grand prix d'Hiver à Milan et Général de Pommeau. Dans Revue de l'Automobile, le chat est incarné par le pilote des rallyes Sébastien Loeb dont l'absence a favorisé l'ascension de Marcus Gronholm et Fords en Turquie. L'Équipe évoque, en utilisant ce dicton, la ligue mondiale et l'absence du capitaine des bleus, Stephane Antiga, le réceptionneur, et des quatre remplaçants. Le Matin prend le soin d'expliciter les références, dès les premières lignes de l'article, et de la façon la plus directe : " C'est connu : quand le chat n'est pas là, ce sont les souris qui dansent. Le chat, dans le motocyclisme suisse moderne, c'est Thomas Luthi (...) ». Non moins directe s'opère, dans l'article, l'identification des souris (ou plutôt, le cas échéant, la souris) « Voilà pour le chat ! La souris ? C'est d'abord ce regard coquin, ce sourire permanent, ces boucles blondes : Dominique Aegerter est un jeune homme heureux $\gg$.

Comme nous le constatons, c'est dans le domaine du sport - que le journal soit spécialisé ou éclectique - que l'on use le plus de l'inachèvement du proverbe. Dans les exemples susmentionnés, l'absence d'une personne est perçue comme une opportunité pour l'épanouissement d'une autre.

Un autre proverbe se trouve souvent cité dans sa version incomplète :

(2) «Qui sème le vent... ». 
Dans un article de La Tribune all Africa (5/1/2004), c'est le FLN (Front de libération algérienne) qui a semé « les graines de la violence de l'État algérien en interdisant la liberté d'expression et de manifestation (ex. l'interdiction de la marche des militants); il ne peut que récolter la haine des citoyens ».

Le livre tragique intitulé « Le vent» de Dorothy Scarborough (1925), adapté au cinéma muet par Victor Sjöström sans grand succès car jugé trop déprimant par la MGM, a eu une sortie retardée et « a été tué dans l'œuf par l'avènement du parlant. Qui sème le vent ...»(Les Échos, 1/7/2004). Le journaliste, Philippe Chevilley, joue sur le fait que le titre du film le condamnait d'avance à l'échec. Qui tourne Le vent (film) est, par analogie, celui qui sème le vent. Les conséquences négatives (échec du film) semblent, aux yeux du journaliste, évidentes, comme si le nom du film suffisait pour l'y prédestiner. Dans le Sud-Ouest, l'article de Pierre-Marie Lemaire (19/4/2004) ayant pour titre «Qui sème le vent » évoque un sujet polémique : l'installation d'éoliennes dans les champs. Le projet de ferme éolienne sur la butte des Moindreaux soulève une tempête de colère et de contestations chez les agriculteurs.

Ainsi, à la lecture du proverbe inachevé, il vient immédiatement à l'esprit du récepteur la suite attendue ainsi que des éléments stéréotypés associés à l'ensemble de l'énoncé proverbial.

Dans certains cas, l'inachèvement du dicton pourrait être justifié par sa prise au sens littéral comme l'illustre l'exemple suivant :

\section{(3) «Quand le vin est tiré »}

Cet énoncé est le titre d'articles parus respectivement dans Libération du 16/4/1999, Le Progrès du 21/11/2003, Le Figaro du 17/11/2004, Le Sud-Ouest du $11 / 12 / 2004$. Dans les articles susmentionnés, il est bel et bien question du breuvage. Cet exemple brave ouvertement tant le critère de métaphoricité 9 que le critère d'opacité sémantique ${ }^{10}$, tous les deux caractéristiques des parémies. La disparition de l'aspect métaphorique empêche une lecture au second degré et justifie également l'intitulé. Le dicton inachevé n'est qu'un prétexte pour faire référence directe au vin.

Dans les exemples de parémies tronquées précédemment envisagées, la transformation reste au niveau syntaxique (et parfois au niveau sémantique comme nous venons de le voir en ce qui concerne la perte du caractère métaphorique ou générique). Or, le détournement à proprement parler doit être en principe un cas de parémie ostensiblement altérée, un cas de défigement formellement marqué: «La forme et le contenu propositionnel du figement sont touchés. Ces modifications permettent un repérage immédiat du défigement même hors contexte » (Lecler, 2006 : 48).

Nous tenterons donc de prouver à quel point le défigement formellement marqué pourrait ouvrir des paradigmes là où, en principe, il n'y en a pas.

Selon Kleiber.

10 Selon Villiers $(2010: 148)$. Notons que condition d'opacité sémantique est facultative et ne s'applique pas à tous les proverbes comme l'ont signalé Kleiber (2010 : 51) et Khojet El Khil (2003 : 140-141). 


\section{Détournement des parémies dans la presse}

De prime abord, nous tenons à préciser que notre travail exclue volontairement les différentes variations qui ne constituent que des variantes attestées (en diachronie ou en synchronie) : Ex. "Qui ne tente rien n'a rien » et sa variante " Qui ne risque rien n'a rien ». Une étude menée par Jean-René Klein, évoque Le Dictionnaire automatique (sous forme de CD-rom) de 1500 proverbes et leurs variantes (2500 variantes environ) depuis le Moyen-âge (2007 : 134) et précise les variations mineures qu'admettent les proverbes : « ajout d'adverbes (jamais, toujours) qui ne compromettent pas la généricité, formes impersonnelles avec ou sans $i l$ (il vaut mieux, vaut mieux...) hésitation il n'est ou il n'y a, entre les éléments de négation, pas ou point, etc. » (Klein, $2007: 153)$.

Si nous écartons les variantes attestées, c'est parce que, lexicalisées et répandues, elles ont perdu leur singularité et témoignent moins de la créativité de leur énonciateur.

Le procédé de réécriture qui entraîne des modifications visibles dans le proverbe est appelé, par la plupart des linguistes français ou francophones " détournement » ou « défigement $»^{11}$. Selon Schapira $(2000: 95)$ « le détournement est, nécessairement :

- soit la déformation, de quelque manière que ce soit, d'un proverbe attesté,

- soit une création originale à partir d'un moule proverbial susceptible d'être immédiatement reconnu et identifié comme tel ».

Pour Schapira, le détournement est un des deux cas de déproverbialisation, l'autre cas étant la négation du message proverbial par la restitution du proverbe au statut de phrase ordinaire pouvant être :

$$
\begin{gathered}
\text { «Il ne trouve pas que + proverbe » } \\
\text { ou } \\
\text { « Je trouve /à mon avis + proverbe à la forme négative ». }
\end{gathered}
$$

Chaque linguiste dresse sa propre liste de procédés qui font qu'un énoncé, où l'on reconnaît l'écho d'un ancien proverbe et qui possède les marques de l'énonciation proverbiale n'appartient pas, pour autant, à l'ensemble des proverbes attestés reconnus et répertoriés dans les dictionnaires. Kleiber (1999: 66) par exemple voit que tout proverbe précédé d'un verbe d'opinion redevient phrase ponctuelle. Grésillon et Maingueneau (1984:115-116) parlent de détournements ludiques en les opposant aux détournements militants, pointant avant tout la valeur perlocutoire attendue de ce type de réécriture. Le détournement s'opère :

1. au niveau des conditions d'emploi et/ou

2. au niveau du signifiant et/ou

3. au niveau du signifié.

${ }_{11}$ Précisons que Charlotte Schapira restreint le détournement aux proverbes attestés seulement et non à n'importe quel énoncé sentencieux. 
Anastassiadis-Symeonidis (2003 : 53-54) s'attaque plutôt au défigement ludique qui, à son sens, est le plus reconnu, et signale uniquement deux procédés : la polysémie/homonymie et la substitution lexicale. Sullet-Nylander (2005:120-121) prend pour terrain d'étude les Unes de Libération et considère le défigement comme simple jeu de mots allant des cas où « le signifiant n'est pas modifié du tout (polysémie) pour aller vers une modification de plus en plus remarquable ${ }^{12}$ paronymie sur deux signifiants ${ }^{13}$, parasynonymes ${ }^{14} \gg$.

Les procédés de détournement sont mieux répertoriés chez Wozniak (1999). Or, la liste des procédés est plus complète chez Barta dans un article publié en deux parties $(2005$; 2006). La première partie recense, en 2005 , la paronomase, la polysémie, l'interprétation du figuré par le littéral, l'homophonie, l'adjonction et les procédés stylistiques (métaplasmes), ces derniers incluant la substitution, l'adjonction, la suppression et la permutation par inversion. En 2006, la suite de son article regroupe d'autres procédés s'inspirant davantage de la stylistique comme les métaphores, les proverbes-valises ${ }^{15}$, les métasémèmes, les métalogismes. La dernière catégorie regroupe les répétitions, les métaphores brisées et les redondances. Toutefois, c'est le classement de Villiers (2010 : 159-162) qui nous semble le mieux fait. Il s'inspire des modèles antérieurs mais il est facile d'accès et moins déroutant car il synthétise les travaux de ses prédécesseurs en évitant les catégories redondantes. Il réduit les procédés à six : la fusion, la substitution, le déplacement, la contraction, l'expansion, la réduction. Il ne manque pas de pointer la possibilité de l'usage de plusieurs procédés de concert. Nous avons déjà étudié plus haut la réduction des parémies - ou leur inachèvement - que nous jugeons comme cas de défigement minime où la manipulation est peu créative et repose sur le bagage linguistico-culturel du récepteur et sa faculté à retrouver la suite attestée de l'énoncé parémique (cas de réduction) qui tantôt garde son caractère générique (cas d'adaptation) ou le perd. Nous focaliserons, dans ce qui suit, sur deux procédés de la réécriture des parémies très fréquents dans la presse : la substitution et l'expansion.

\subsection{Détournement par substitution}

La substitution est le procédé vedette du détournement. " Cette opération consiste à remplacer une unité du proverbe initial par une autre unité, essentiellement de même nature » (Wozniak, 2009 : 190). La substitution peut être phonétique, lexicale ou syntaxique.

\subsubsection{Substitution fondée sur le signifiant}

La parenté phonétique s'avère être une ligne de force, étant donné que la modification porte sur le signifiant, pour entraîner, conséquemment, celle du signifié. Plus la modification est minimale, plus elle est éloquente et plus elle attire l'attention du récepteur sur le jeu de mots. C'est ainsi que la voyelle [ø] est supplanté par la voyelle [u] dans

\footnotetext{
Cas d'homophonie ou cas de paronymie sur un signifiant (homophones à un phonème près).

Mots-valises.

Simples proximités sémantiques.

Combinaison de plusieurs unités phraséologiques. Nous avons «des proverbes-valises contenant des proverbes uniquement et proverbes-valises contenant un proverbe au moins et une locution" (Barta, $2006: 64$ ).
} 
(4) «Un homme averti en Vaudou» (Midi Libre, 16/11/2007).

« Vaut deux » et «Vaudou » sont, phonétiquement parlant, très proches. Les rites vaudou sont mentionnés ici pour faire écho aux pratiques de sorcelleries que subissent certaines Africaines exploitées par des marabouts les gardant sous leur empire afin de les entraîner dans des réseaux de prostitution. Ici, la parémie modifiée est plutôt à enjeu ludique ${ }^{16}$. La preuve en est bien la suivante : la parémie-mère (Un homme averti en vaut deux) reste celle visée principalement par le journaliste ; la parémie altérée n'est qu'un faux masque forgé par complicité pour faire écho à la thématique de l'article de presse. Or, la visée pragmatique de « sensibilisation » de ce texte journalistique rejoint celle du proverbe initial. Dans l'exemple de parémie-cible susmentionnée, la paronymie affecte un seul élément du signifiant, un phonème. L'effet de détournement est réussi et reste facilement identifiable.

(5) « À chaque jour suffit sa haine ou sa peine ?»

La parémie d'origine biblique se trouve altérée au profit du profane. Sans trop modifier les sonorités, nous lisons dans Le Maine Libre du 24 novembre 2012 «A l'UMP, à chaque jour suffit sa haine », article paru pour évoquer le duel politique, la tension entre Fillon et Coppé. L'énoncé «A chaque jour suffit sa peine » revêt un habillage nouveau grâce à la suppression du son [p] de la « peine » qui devient « haine », deux paronymes complètement éloignés sur le plan de l'orthographe. Cette forme modifiée n'est ni singulière ni unique : nous la retrouvons dans African Manager, dans un article de Khaled Boumiza intitulé « Il y a en Tunisie au moins 2 millions de koffar, mécréants » (20/12/2012) : « Mme Mansour n’a certainement pas bien lu le Saint Coran et ce qu'il dit à propos de l'insulte de l'autre. Elle n'a pas non plus bien écouté les paroles du Cheikh Ghannouchi sur le takfir ${ }^{17}$. Elle ferait bien donc de réviser ses leçons. À chaque jour, suffit sa haine ! ».

Il convient de mentionner au passage que cette forme modifiée remonte à 2011 où elle connaît un essor avec le chanteur François Ville dans son titre musical « A chaque jour suffit sa Haine », haine avec grand $\mathrm{H}$, où il traite la violence, le racisme, les bains de sang qui menacent le monde.

Dans (6a), il s'agit d'un énoncé extrait d'un article sur les déguisements vestimentaires dans l'Histoire, où l'on emprunte l'habit de l'autre sexe afin de se déguiser ou de changer d'identité.

(6a) «L'habit ne fait pas le moi » (Titre, Libération, 21/6/2001).

Jusqu'ici, la substitution est minimale et ne touche que les phonèmes.

16 De fait, « il y a détournement ludique lorsque ce détournement entre dans le cadre des purs jeux de mots, et n'est pas au service d'un enjeu idéologique, politique... » (Grésillon et Maingueneau, 1984 : 114). Il s'oppose au détournement militant.

17 Kufr (arabe رفك: ريفكت $1 \mathrm{kufr}$ ) signifie mécréance et takfir le fait de considérer une personne comme non-croyante. 


\subsubsection{Substitution lexicale}

Lorsqu'elle altère des unités supérieures (le lexique), la substitution exige plus de calculs interprétatifs de la part du récepteur. Aucune parenté phonétique n'est perçue entre le substituant et le substitué. Par exemple, le proverbe « L'habit ne fait pas le moine », par substitution lexicale devient le titre d'un article.

(6b) «L'habit ne fait pas la karateka » (titre dans Reuters- les actualités en français, 24/5/2001)

Dans (6b), il ne s'agit que d'une championne de Karaté qui, agressée par des violeurs, les a mis au tapis. Le changement du signifiant peut entraîner le changement de sémantisme de la parémie comme le fait la correspondante de Libération, Claude Guibal, en écrivant dans son article sur la danse orientale intitulé « le Caire nombril du monde » :

(6c) «L'habit ne fait pas la danseuse » (Libération, 23/11/2001)

pour dire que c'est la performance de la danseuse et non le costume échancré qui fait la notoriété de l'artiste.

Inspiré des fameuses voies romaines et signifiant qu'il est possible d'atteindre le même but par plusieurs moyens, l'énoncé parémique « tous les chemins mènent à Rome » est parmi les plus détournés par substitution. Pour viser une destination précise, il suffit de mettre un toponyme à la place de Rome et le défigement se réalise :

(7a) « Tous les chemins mènent à Nassau, capitale des Bahamas a-t-elle lancé » (L’Agéfi, 30/10/1996)

(7b) « Car en France, en revanche, rien de nouveau. Tous les chemins mènent toujours à Paris $»^{18}$ (Les Échos, 18/11/1998).

$\mathrm{Au} \mathrm{XII}^{\mathrm{e}}$ siècle, dans une acception chrétienne, Rome était le siège du pape. Et l'on dit que la parémie affirmait dans le temps que, malgré les difficultés, on peut accéder à Dieu ou encore que toute méditation conduit à l'existence du bon Dieu créateur de l'Univers. Cette trace religieuse, nous la retrouvons dans le détournement suivant $(7 \mathrm{c})$ où il est question du pèlerinage breton :

(7c) « Tous les chemins mènent au Tro Breiz » (titre, Libération 12/08/1998).

(8) « Rira bien qui rira le premier»

L'exemple (8) apparait respectivement comme titre dans L'Est Républicain (13/10/2008), Le Progrès (23/11/2009), La Tribune de Genève (16/2/2008). Ceci a pour effet, bien entendu, de bouleverser complètement le sémantisme de la parémie initiale. Ce proverbe détourné figure également dans un article d'Ouest France

18 Pour parler de la centralisation des pouvoirs et institutions. 
(supplément, 10/9/2008) où il renvoie au festival de l'humour à Cossé-le-Vivien. Le rôle du défigement se restreint à faire allusion au comique et à initier le public à s'empresser pour assister « en premier » à ce festival.

\subsubsection{Substitution syntaxique}

Plus la substitution prend de l'ampleur, plus le défi que se propose le journaliste est important : comment ne pas rater sa cible à la fois sémantico-pragmatique et stylistique tout en effectuant des changements de plus en plus tangibles sur le plan syntaxique ? Examinons les exemples ci-dessous qui travestissent le proverbe « $\mathrm{Au}$ pays des aveugles, le borgne est roi » :

(9a) " $\mathrm{Au}$ pays des aveugles, la boussole est reine »(Titre, Libération, $12 / 10 / 2001)$

(9b) «Au pays des aveugles, le cinéma s'égare » (Titre, Le Temps, 8/10/2008)

(9c) «Au pays des aveugles il n'y a que 'l'œil du web' qui puisse être roi » (Titre, L'Yvonne républicaine-sud, 30/10/2010)

(9d) «Au pays des aveugles, le borgne fait führer » (Libération 26/4/2002)

Le moule du proverbe est plus ou moins préservé dans les exemples (9a), (9b) et (9d) qui gardent l'empreinte de la syntaxe du figement par la ponctuation (la virgule) qui scinde en deux parties le proverbe. L'exemple (9c) s'écarte du patron formel par l'allongement du remplaçant. Tous les exemples convoquent l'actualisation de l'isotopie qui fait appel au sens de la parémie-mère (Au pays des aveugles, le borgne est roi). Le procédé de décodage opère comme suit : le récepteur perçoit le début du proverbe. Sans continuer la lecture, il anticipe sur la suite connue dans un premier temps, puis, dans un second temps, il rectifie sa conception par rapport aux nouvelles propositions modifiées données par les journalistes et par rapport au contexte d'emploi.

Nous avons également constaté que les substitutions au niveau phrastique sont à attendre plutôt dans les parémies à structure binaire :

(10) « Noël au balcon, choux-fleurs à foison » (Titre de l'article, Libération, $15 / 4 / 1998)$

où il s'agit de l'abondance des choux-fleurs à cause de l'hiver doux au point de subsister jusqu'au mois d'avril.

(11) « Sans le chat, les souris dépriment ! » (La tribune de Genève, 16/2/2008)

L'exemple (11) figure dans un l'article de presse où le journaliste crée une longue allégorie : le joueur de tennis Tsonga est assimilé au chat (" notre super matou »), qui « ronronne » sans trop se préoccuper de la progression inexorable de ses deux « rivaux » (« le pitbull de Manacor ${ }^{19}$ et le pitre de Belgrade $\left.{ }^{20} »\right)$, qui au dire du jour- 
naliste, sont assimilés, à leur tour, à la fin de l'article, à « deux siamois de service ». Vaincu à Melbourne, Tsonga est aussi « l'échaudé [qui] craint l'eau froide », selon les experts de tennis comme le précise le journaliste.

A bien considérer, dans les exemples étudiés, les parémies originelles ne perdent pas leur notoriété. La presse les recycle pour réutiliser de manière opératoire la forme nouvelle tout en invitant à la reconstruction de la parémie initiale dans sa forme canonique. Ce qui compte, en premier chef, est la visée communicative du journaliste, l'effet perlocutoire qu'il cherche à produire grâce à la réécriture-recomposition de la parémie-mère.

\subsection{Détournement non marqué : l'expansion}

Entre l'inachèvement et le défigement à proprement parler (par substitution), nous placerons l'expansion qui, selon Villiers (2010:160), consiste à « ajouter un ou plusieurs éléments au proverbe cible » : une lettre, un mot ou une proposition. À vrai dire, le proverbe lui-même n'est pas formellement altéré. C'est le co-texte suivant (ou la suite ajoutée) qui défige la parémie. À notre sens, la parémie est à la fois autre et même. Lecler (2006 : 48) parle de « défigement non marqué ».

Le défigement non marqué formellement, comme son nom l'indique, ne présente pas de modifications formelles (ou des modifications minimes) par rapport au figement. Il a ainsi l'apparence de l'expression figée. L'énoncé coupé de son contexte ne comporte pas d'éléments susceptibles d'indiquer qu'il s'agit en effet d'un défigement : le contenu prépositionnel ne semble pas modifié. Seul le recours à la situation de communication et au contexte permet de percevoir une autre construction du sens.

Aussi pouvons-nous lire :

(12) « L'habit ne fait pas le moine... ni le républicain » (Titre, Libération $4 / 10 / 2000)$

(13) «Il faut battre le fer quand il est chaud, et la crème quand elle est fraîche » (Le Figaro, 9/10/2001)

(14) «Quand le chat n'est pas là ou qu'il dort sous un palmier, les souris dansent » (Le Matin, 1/12/2007)

Ce qui nous concerne ici, c'est l'émergence d'une nouvelle construction par l'adjonction d'éléments supplémentaires à l'énoncé d'origine. En (12) et (13), l'expansion suit la parémie. En (12), l'ajout altère une des caractéristiques fondamentales du dicton : il lui ôte son caractère générique. Dans la parémie-mère, le mot « habit » qui est à prendre dans le sens large renvoyant à toute apparence, vestimentaire ou comportementale. Le mot « moine » est emblématique de toute personne dont la tenue reflète ses croyances, ses idéologies. L'ajout de " ni le républicain » suggère qu'il faut se méfier des Républicains qui feignent de l'être. Le dicton en (13) est souvent utilisé pour faire allusion à l'importance de saisir le moment propice. L'expansion 
vient renforcer cette idée tout en ajoutant une nuance sémantique intéressante : si le bon moment pour battre le fer est lorsqu'il est chauffé, la crème est à battre lorsqu'elle est fraiche. Ceci dit, il faut tenir compte du fait que ce qui convient au fer peut ne pas convenir aux autres éléments. Toutefois, quelle que soit la situation, il faut être assez habile pour choisir le moment d'agir et ne pas laisser trainer ses tâches. En (14), l'expansion figure en position médiane, et par conséquent, brise la structure rythmique de la forme initiale au profit de l'introduction d'une précision supplémentaire : l'absence du chat (ou de toute autre autorité redoutable) n'est pas nécessairement une absence physique. Elle peut être perçue comme un manque d'action, inertie temporaire ou passivité.

\section{Phraséodidactique du (dé-)figement :}

En tant que branche de la linguistique « placée à mi-chemin entre le lexique et la syntaxe, la phraséologie s'occupe des signes polylexicaux composés d'au moins deux mots, stables, répétés et souvent figurés.» (González-Rey, 2008b : 5). Lorsqu'elle s’associe à la didactique, la phraséologie donne naissance à la phraséodidactique, « ou didactique de la phraséologie, (qui) concerne l'enseignement/apprentissage des expressions figées, dans le cadre de l'acquisition des langues vivantes, que ce soient des langues maternelles ou des langues étrangères. Cette discipline se définit non seulement par son objet (les propriétés formelles, sémantiques et pragmatiques des unités phraséologiques) mais aussi par la fonction qu'elle réclame au sein de la didactique des langues étrangères puisque la présence de la phraséologie y est quasiment inexistante ». De ce fait, elle revendique un espace particulier pour défendre « la mise en place d'un module propre visant l'acquisition chez l'apprenant d'une compétence active en expressions figées » (González-Rey, 2008a : 1). À noter que l'intérêt consacré à l'enseignement des séquences figées est faible ${ }^{21}$ : les apprendre par cœur, avec leurs significations et leurs contextes d'emploi, demeure la pratique la plus courante dans les cours de langue. Souvent, il s'agit d'exercices lacunaires à compléter à l'aide d'un lexique proposé ou des parties de proverbes à associer. Certaines activités présentent des proverbes en désordre à reconstruire. Leur objectif est le même : la recomposition du proverbe afin de faciliter par la suite sa mémorisation. D'autres exercices vont un peu plus loin en proposant d'associer un scénario ou une histoire au proverbe qui convient ${ }^{22}$.

Généralement, en matière de didactique des langues étrangères, le problème qui se pose, en abordant les proverbes, est celui de la compréhension : " Les expressions figées passent souvent inaperçues aux yeux des locuteurs natifs alors qu'elles

21 González-Rey dénombre (2008a) quelques ouvrages de didactique du français langue étrangère, parus aux Presses Universitaires de Grenoble (PUG), ouvrages qui consacrent des activités aux séquences figées : Le chemin des mots, pour un apprentissage méthodique du vocabulaire français, de Danièle Dumarest et Marie-Hélène Morsel (2004) ; Émotions-Sentiments, nouvelle approche lexicale du FLE, de Cristelle Cavalla et Elsa Crozier (2005) et Dites-moi un peu, méthode pratique du français oral d'Anne-Marie Hingue et Karine Ulm (2005). Le seul manuel propre à la didactique des expressions figées est intitulé Phraseotext - Le Français Idiomatique (2015) d'Isabel González-Rey, Vilmos Bárdosi, Jean-Louis Dufays, et al. destiné aux débutants, grands adolescents ou adultes. Santiago : Universidade de Satiago de Compotela. Cette méthode est intégrée à l'ouvrage d'Isabel GONZÁLEZ REY, (2007) La didactique du français idiomatique. Cortil-Wodon, Belgique : E.M.E.

22 Voir les activités sur le site http://www.bonjourdumonde.com/blog/grece/9/fiches-pedagogiques/les-proverbesen-classe-de-fle-fiche-pedagogique-2, Publié le 28 mai 2013, consulté le 7 mars 2017. 
sont vite repérées par les étudiants étrangers. Leur opacité représente un écueil dans l'apprentissage des langues étrangères et amène souvent les élèves à les ignorer, ce qui les éloigne de fait d'une compétence complète de la langue cible » (Sułkowska, 2013 : 9). Cela étant, le scénario pédagogique classique ressemblerait, à notre sens, à ce qui suit : entraîner de prime abord l'apprenant à identifier la parémie (ce qui n'est pas toujours évident) et à l'appréhender globalement, c'est-à-dire en tant qu'un ensemble et non pas en tant qu'éléments pris séparément. Ensuite, l'apprenant devrait passer du sens littéral à la compréhension du langage figuré. Dans cet ordre d'idée, Rius-Dalmau (2014 : 337) propose ce qui suit :

Nous pensons utile de les classer selon leur degré de transparence/opacité. On considère l'expression figée transparente si les locuteurs reconnaissent la forme et le sens de chaque élément. Dans les cas contraires, l'unité est opaque. Ainsi, dans des étapes initiales, l'enseignant devra commencer par présenter les formes fixes dont la signification est transparente, le sens littéral domine. Voilà pourquoi nous proposons une démarche didactique allant de la transparence à l'opacité.

Une fois la parémie saisie, l'étape suivante est la recherche d'une traduction ou plutôt d'un équivalent dans la langue maternelle de l'apprenant. Telle semble être la consigne la plus répandue en matière d'activité interculturelle et de perspectives contrastives.

Certains enseignants-chercheurs font des parémies une activité ludique, un jeu comme Mireille Grosjean qui, dans son article « Le jeu : une idée de plaisir » publiée dans la revue Babylone (2003 : 54) propose, dans une perspective toujours contrastive, un domino des proverbes :

Les élèves reçoivent des cartes où figurent deux proverbes, proverbe $\mathrm{x}$ en langue $A$, proverbe $y$ en langue $A$; autre carte : proverbe $m$ en langue $B$, proverbe $n$ en langue $\mathrm{B}$, etc., jusqu'au proverbe 38 . À côté du proverbe y en langue B, on mettra la carte suivante qui comporte le proverbe y en langue A et un autre proverbe en langue A également. De nouveau, on tentera de trouver son correspondant en langue B et ainsi de suite. Dans "Échanges de classes clé en main", on trouve 38 proverbes dans leurs versions allemande et française. Il s'agira donc de regrouper « Ende gut, alles gut » avec « Tout est bien qui finit bien » par exemple ».

L'auteure de l'article part de l'introduction des proverbes ayant à leur tour des libellés dans les deux langues pour appréhender ensuite les proverbes que l'on ne retrouve pas dans la langue apprise ou inversement. Ces suggestions sont intéressantes sur le plan culturel mais ne rendent pas compte de la particularité phraséologique des parémies françaises. Nous pensons avec Théophanous (2007 : 118) que «plusieurs possibilités de travail s'offrent sur le plan syntaxique basées sur le repérage, le classement, la reformulation ou la paraphrase ».

Pour faire le lien entre recherche en sciences du langage et en phraséodidactique du Français, nous viserons à débloquer la parémie (modifier certaines de ses propriétés) en vue d'obtenir de nouvelles formulations qui s'écartent (formellement et/ou sémantiquement) de la parémie-mère. Dans quel objectif? Travailler le défigement pour mieux comprendre le figement, enrichir le bagage lexical des apprenants et développer la reformulation. Nous exposerons quelques pistes que nous avons ex- 
plorées avec une vingtaine d'apprenants universitaires (niveau B2), âgés de 19-20 ans et dont le français était aussi une langue de scolarisation.

\subsection{Construction du savoir}

L'apprenant se construit des représentations personnelles du fonctionnement de la langue (sa propre vision). Le rôle de l'enseignant est de nourrir cette activité épilinguistique $^{23}$ dans un premier temps, puis de déséquilibrer un système en formation au moyen de certaines activités pédagogiques, dans un deuxième temps.

Pour construire et consolider le savoir des apprenants sur les parémies, une séance préliminaire est consacrée à la découverte des spécificités des proverbes. Suite à un remue-méninge, les apprenants arrivent à retrouver seuls quelques caractéristiques et les formulent dans leur propre métalangage comme suit :

- formule de sagesse populaire.

- formule générique, applicable en principe en tout lieu, en toute époque.

- Style imagé/figuré.

- Formule souvent concise.

- prosodie particulière (phrases rythmées, jeux de mots et de sonorités, etc.).

- Syntaxe particulière dans la majorité des cas (le sujet peut être un infinitif, une relative nominale; absence de déterminants ou article zéro ; ellipse du verbe, emploi du présent de l'indicatif etc.).

Pour donner aux apprenants une consolidation à ces connaissances partagées sur les parémies, ils seront exposés aux pages des dictionnaires monolingues (ex. Le dictionnaire Larousse) consacrées aux proverbes, à quelques dictionnaires et ouvrages ${ }^{24}$ entièrement consacrés à l'étude des proverbes.

Au cours de la deuxième séance, nous faisons défiler, comme à la parade, les articles de presses avec les proverbes et dictons détournés. Il s'agit bel et bien d'une phase où nous cherchons intentionnellement à déstabiliser ce que l'étudiant a pu constater lors des séances antérieures (quant au figement des proverbes) afin de l'amener à conclure que le figement n'est pas absolu et à retrouver un nouvel équilibre dans la construction de savoir. En outre, cette séance a pour objectif l'élaboration d'hypothèses, de la part des apprenants, sur les diverses modifications (phonétique, lexicale, syntaxique) que subissent les parémies et sur les effets du défigement ${ }^{25}$.

\subsection{Phase de production}

La troisième séance est centrée sur la production dans une pédagogie active ne correspondant pas seulement à un ensemble d'outils prêts à l'emploi, mais à la mise en place d'activités susceptibles de provoquer la dynamique mentale des apprenants.

23. Les psycholinguistes qualifient ainsi une activité qui s'apparente à un comportement métalinguistique mais qui n’engage pas de la part du locuteur un savoir sur la langue ni des procédures de travail conscientes.

(Dournon, 1986 ; Maloux, 1995 ; Desallamand, et Stalloni, 2010).

25 Il est conseillé d'aborder quelques exemples de variantes synchroniques au début de cette séance pour aménager le terrain avant de passer au détournement à proprement parler. 
Une des consignes qui pourraient être travaillées consiste à donner une version tronquée d'un énoncé proverbial connu et à inviter les apprenants, dans un premier temps, à fournir la suite attendue. Un exercice simple et classique mais qui prépare les étudiants à l'idée d'anticipation que l'on doit appliquer lorsqu'on lit en général. Même si les versions proposées en réponse sont des variantes de la parémie initiale, elles sont en principe acceptées. Dans un deuxième temps, une consigne de réécriture sera donnée aux apprenants afin de les encourager à produire, sous-contraintes (consignes spécifiques) ou librement. Une liste de proverbes et dictons est donnée aux apprenants avec leur sens bien indiqué. La première tâche de l'étudiant est de les réécrire en changeant (partiellement ou complètement) leur forme mais non pas leur sens. Il s'agit là de captation ${ }^{26}$. Voici quelques exemples de productions d'apprenants :

\begin{tabular}{|c|c|}
\hline Parémie & Réécriture sans changement sémantique \\
\hline (15) Un homme averti en vaut deux & $\begin{array}{l}\text { (15a) Une personne avertie en vaut deux } \\
\text { (15b) Un ennemi averti en vaut deux } \\
\text { (15c) Une femme avertie en vaut quatre } \\
\text { (15d) Une armée avertie en vaut deux }\end{array}$ \\
\hline $\begin{array}{l}\text { (16) C'est en forgeant qu'on devient forge- } \\
\text { ron }\end{array}$ & $\begin{array}{l}\text { (16a) C'est en étudiant qu'on devient étu- } \\
\text { diant } \\
\text { (16b) C'est en étudiant qu'on devient intel- } \\
\text { ligent } \\
(16 \mathrm{c}) \text { C'est en dansant qu'on devient dan- } \\
\text { seur } \\
\text { (16d) C'est en pratiquant qu'on devient } \\
\text { professionnel. } \\
\text { (16e) C'est en trichant qu'on devient tri- } \\
\text { cheur*. } \\
\text { (16f) C'est en travaillant qu'on devient ex- } \\
\text { cellent. } \\
\text { (16g) C'est en mendiant qu'on devient } \\
\text { riche*. }\end{array}$ \\
\hline (17) La nuit tous les chats sont gris & $\begin{array}{l}\text { (17a) La nuit, toutes les femmes sont pa- } \\
\text { reilles. } \\
\text { (17b) La nuit, toutes les ombres sont } \\
\text { sombres. } \\
\text { (17c) La nuit, tous les chemins sont sem- } \\
\text { blables. } \\
\text { (17d) La nuit, tout se ressemble. } \\
\text { (17e) La nuit, le ciel est gris*. } \\
\text { (17f) La nuit, tous les gens sont au lit*. } \\
\text { (17g) Le matin, toutes les fleurs sont épa- } \\
\text { nouies*. }\end{array}$ \\
\hline
\end{tabular}

26 « La captation consiste à détourner en allant au maximum dans le sens de la structure sémantique ainsi exploitée » (Grésillon et Maingueneau, 1984 :115). 
(18) Tous les chemins mènent à Rome
(18a) Toutes les rues conduisent à la mai- son*
(18b) Toutes les décisions conduisent au destin.
(18c) Tous les chemins mènent à la vérité.
(18d) Toutes les tentatives mènent à la vic- toire*.
(18e) Tous les chemins mènent au destin.
$(18 \mathrm{~g})$ Toutes les cigarettes mènent à la mort.

Après une mise en commun, une halte analytique des propositions est faite par l'enseignant pour explorer l'originalité de ces productions et le processus de défigement mis en œuvre. Dans la majorité des productions citées, la stabilité sémantique de la parémie-mère est grosso modo maintenue ${ }^{27}$ ce qui explique leur réussite. Toutefois, certaines propositions nous paraissent choquantes parce qu'elles contredisent l'éthique (16g), qu'elles sont banales (17f), qu'elles divulguent une vérité générale $(17 \mathrm{~g})$, ou qu'elles représentent une généralisation hâtive et non fondée (18a) et (18d). Dans les réécritures de la parémie (15), la prudence est mère de sûreté : les substitutions lexicales faites par les apprenants sont minimes (modification d'un seul mot) hormis en $(15 \mathrm{c})$ où l'apprenant a changé deux termes. Dans la réécriture des autres proverbes et dictons (16) à (18), les apprenants se sont montrés plus hardis.

Cet exercice a pour objectif l'acquisition lexicale, certes, mais aussi l'apprentissage de la formulation et la reformulation. À noter que les reformulations des étudiants préservent parfois, outre le signifié, la matrice syntaxique. Toutefois, c'est la métaphoricité et la musicalité qui sont souvent difficiles à maintenir intactes. Les apprenants les plus « futés », les plus « doués » ou les plus prodiges ont, soit préservé la musicalité de l'énoncé parémique initial (17e), soit inventé des rimes internes là où il n'y en avait pas (par comparaison au proverbe ou dicton de départ) $(17 \mathrm{~b}, 17 \mathrm{f}, 18 \mathrm{e})$.

La deuxième activité que nous proposons s'apparente plutôt au pastiche et à l'écriture d'invention, c'est-à-dire écrire à la manière de., qui est, comme le signale Fumaroli (2012), « une forme légale et créatrice de l'imitation $»^{28}$. Autrement dit, les étudiants sont invités à garder la forme prosodiquo-syntaxique du proverbe tout en changeant son sens. L'exploration par le pastiche des diverses formes d'énoncés parémiques donne à voir le savoir-faire stylistique des apprenants, les tâtonnements poétiques. Il s'agit de cas de subversion ${ }^{29}$ : il y aura donc, parmi ces tentatives, à la fois des substitutions lexicales et une originalité sémantique. Les modifications effectuées témoignent de la polysémie d'une même construction phrastique, d'un même moulage. Aussi avons-nous obtenu les productions réussies suivantes :

27 Les exemples de productions avec astérisque contiennent de légères déviances sémantiques ou des nuances péjoratives qui n'existent pas dans le sémantisme du proverbe originel.

28 Titre de la préface du volume 112 de la Revue d'histoire littéraire de la France.

29 « La subversion cherche (...) à faire apparaître une contradiction entre le sens véhiculé par l'énonciation de la structure originelle (notée E0) et celui de l'énonciation de la structure résultant du détournement (notée E1) » (Grésillon et Maingueneau, $1984: 115$ ). 


\begin{tabular}{|l|l|}
\hline \multicolumn{1}{|c|}{ Parémies } & \multicolumn{1}{|c|}{ Réécriture avec changement sémantique } \\
\hline (19) Qui va à la chasse perd sa place. & $\begin{array}{l}\text { (19a) Qui va à la gare, oublie ses cauchemars. } \\
(19 \mathrm{~b}) \text { Qui dort à la maison, perd ses ambitions. } \\
(19 \mathrm{c}) \text { Qui va à la classe, perd sa tasse. } \\
(19 \mathrm{~d}) \text { Qui va au magasin, achète des mocassins. }\end{array}$ \\
\hline $\begin{array}{l}\text { (20) Rira bien qui rira le dernier. } \\
\text { (21) Au royaume des aveugles, le Mangera bien qui mangera le premier. } \\
\text { borgne est roi. }\end{array}$ & $\begin{array}{l}\text { (21a) Au royaume des animaux, le lion est roi. } \\
(21 \mathrm{~b}) \text { Au royaume des athées, le pécheur a tous } \\
\text { les droits. } \\
(21 \mathrm{c}) \text { Au royaume des corrompus, le voleur fait } \\
\text { loi. }\end{array}$ \\
\hline
\end{tabular}

Au dire de Grésillon et Maingueneau (1984), « un proverbe peut en cacher un autre $»^{30}$. Dans les exemples ci-dessus, la créativité réside dans la transparence des productions : malgré les maintes transformations lexicales et sémantiques, nous ne perdons pas de vue la parémie initiale. Grâce à l'air de la chanson, on retrouve les paroles, et par analogie, grâce au moule très connu, on retrouve la forme figée du proverbe originel cachée sous ce travestissement, sous ce détournement ludique. Les apprenants mémorisent de la sorte plus facilement la parémie-mère, et parallèlement, connaissent le plaisir d'inventer, de créer.

\section{Conclusion}

Dans la présente recherche, nous avons abordé quelques exemples de détournements des parémies en contexte médiatique et la réexploitation de ces procédés en contexte didactique. L'inachèvement des parémies, ce non finito par choix ${ }^{31}$, sollicite, comme nous l'avons remarqué, la culture du lecteur et son bagage de connaissance en matière de sagesse populaire dans une sorte de pacte tacite où le lecteur devient coopératif. L'invitation à la reconstruction du modèle proverbial initial, dans sa forme canonique et complète, est bien présente. La suite attendue (l'élément in absentia), comme nous l'avons vu, est souvent à remplacer par un éventail de possibilités dictées par le contexte médiatique. Si la troncation de l'énoncé parémique le dote d'un changement probable, le détournement, quant à lui, assure indubitablement un changement au niveau formel et/ ou sémantique. Que le détournement porte sur le signifiant et/ou le signifié et/ ou l'emploi-même du proverbe, il s'agit, comme nous l'avons constaté, de stratégies stylistique et communicative astucieuses et chargées d'effets.

Nous avons ciblé, dans une perspective phraséodidactique appliquée, l'exploitation du défigement. Nous espérons avoir atteint par là le but que nous nous sommes fixé au départ : offrir un scénario pédagogique visant une didactisation potentielle

$30 \quad$ Titre de leur article paru dans Langages $\mathrm{n}^{\circ} 73$ (1984).

31 En art, l'inachèvement volontaire ou par choix s'oppose à l'inachèvement nécessaire (la difficulté de réaliser la tâche vue son ampleur ou la fatigue de l'artiste) ou l'inachèvement par impossibilité (incompatibilité entre la matière ou le matériel utilisé par l'artiste et son projet d'œuvre). 
du défigement - qui dépasse l'exercice classique de la mémorisation des parémies afin de provoquer la dynamique mentale des apprenants et de leur faire savourer le plaisir d'inventer ou de créer (côté créativo-ludique concomitant de l'apprentissage). Ce faisant, les étudiants deviennent plus actifs, et par conséquent, favorables au réinvestissement des savoirs appris.

\section{Références bibliographiques}

Anastassiadis-Symeonidis, A., (2003) "Que peut-il arriver à une expression figée ? », in Cahiers de lexicologie et de lexicographie. $\mathrm{N}^{\circ} 82$, éd. Honoré Champion, pp. 51-59.

Anscombre, J.-C., (1989) « Théorie de l'argumentation, topoï, et structuration discursive », in Revue québécoise de linguistique. Vol. 18, n 1, pp. 13-56.

Anscombre, J.-C., (1994) « Proverbes et formes proverbiales : valeur évidentielle et argumentative », in Langue française. $\mathrm{N}^{\circ} 102$, pp. 95-107.

Bally, Ch., (1905) Précis de stylistique, Esquisse d'une méthode fondée sur l'étude du français, Chapitre IV. Genève, Eggimann,

Barta, P., (2005) «Au pays des proverbes, les détournements sont rois. Contribution à l'étude des proverbes détournés du français (I) », in Paremia. No 14 , pp. 139-152.

Barta, P., (2006) «Au pays des proverbes, les détournements sont rois. Contribution à l'étude des proverbes détournés du français (II) », in Paremia. $\mathrm{N}^{\circ} 15$, pp. 57-71.

Connena, M., (1988) «Sur un lexique-grammaire comparé de proverbes», in Langages. Vol. $23, \mathrm{n}^{\circ} 90$ («Les expressions figées »), pp. 99-116

Connena, M., (2002) « Sur l'historique des proverbes », in Maiello, G. \& R. Stajano (dir.), Collage. Studi in memoria di Franca Caldari Bevilacqua, Salerno/Milano, Oedipus, pp. 35-51.

Desalmand, P. \& Y. Stalloni, (2010) 365 proverbes expliqués. Paris, Chêne.

Dournon, J.-Y., (1986) Le dictionnaire des proverbes et dictons de France. Paris, Hachette.

Fiala, P., (1987) « Pour une approche discursive de la phraséologie - Remarques en vrac sur la locutionalité et quelques points de vue qui s'y rapportent, sans doute », in Langage et société. $\mathrm{N}^{\circ} 42$, pp. 27-44.

Fournet-Pérot, S., (2013) « Les proverbes au didactisme pluriel : description, usage et raison d'être », in Paremia. N²2, pp. 187-197.

Fumaroli, M., (2012) « Une forme légale et créatrice de l'imitation : le pastiche », in Revue d'histoire littéraire de la France. Vol. 112, nº 1, pp. 3-6.

Gonzalez-Rey, I., (2008a) «La phraséodidactique en action. Les expressions figées comme objet d'enseignement », in La culture de l'autre : l'enseignement des langues à l'Université : Deuxième Rencontre Hispano-français de Chercheurs (SHF/APFUE) [En ligne], École Normale Supérieure Lettres et Sciences Humaines (26 au 29 novembre 2008). Disponible sur :_https://dialnet.unirioja.es/descarga/articulo/4026980.pdf [Dernier accès le 24 septembre 2017].

Gonzalez-Rey, I., (2008b) La didactique du français idiomatique. Fernelmont (Belgique), E.M.E.

Grésillon, A. \& D. Maingueneau, (1984) « Polyphonie, proverbe et détournement, ou un proverbe peut en cacher un autre », in Langages. Vol. 19, $\mathrm{n}^{\circ} 73$, pp. 112-125.

Gross, M., (1988) « Les limites de la phrase figée », in Langages. N 90, pp. 7-22.

Grosjean, M., (2003) « Le jeu : une idée de plaisir » in Babylone. $\mathrm{N}^{\circ} 1$, pp. 54-57. 
Ibrahim, A. H., (1998) "Constructions figées et constructions à supports ", in Mejri S. (et al.), Le figment lexical. Tunis, CERES, pp. 373-386.

Khojet El Khil, N., (2003) «Transparence sémantique, traduction et figement syntactique dans les locutions verbales », in Mejri, S. (et al.) (éds.), Traduire la langue, traduire la culture. Éd. Maisonneuve \& Larose, coll. Lettres du sud, pp. 137-146.

Kleiber, G., (1989) «Sur la définition du proverbe », in Greciano, G. (éd.), Europhras 88, Phraséologie contrastive. Strasbourg, Actes du colloque international, Université des Sciences Humaines de Strasbourg, Collection Recherches Germaniques, 2, pp. 233-252.

Kleiber, G., (1999) «Les proverbes : des dénominations d'un type très très spécial », in Langue française. $\mathrm{N}^{\circ} 123$, pp. 52-69.

Kleiber, G., (2000) « Sur le sens des proverbes », in Langages. N¹39, pp. 39-58.

Kleiber, G., (2009). « Sémantique proverbiale : proverbe, dénomination et métaphore », in Iliescu, M., Danler, P. \& H. Siller (Eds), Actes du XXVe Congrès International de Linguistique et de Philologie Romanes. Berlin, De Gruyter, pp. 19-46.

Kleiber, G., (2010) «La métaphore dans les proverbes : un trait définitoire ou non ? », in (Pré)-Publications. Numéro spécial 196, pp. 41-62.

Klein, J.-R., (2007) « Le figement dans les proverbes et les expressions verbales figées : un débat qui n'est pas encore... figé », in Conde Tarrío, G. (éd.), Nouveaux apports à l'étude des expressions figées. Bruxelles, Éd. E.M.E. et Intercommunications, pp. $129-156$.

Lecler, A., (2006) « Le défigement : un nouvel indicateur des marques du figement ? », in Cahiers de praxématique. №46, Montpellier, Pulm, pp. 43-60.

Maloux, M., (1995) Dictionnaire des proverbes, sentences et maximes. Paris, Larousse.

Mejri, S., (2008) «Inférence et structuration des énoncés proverbiaux», in Leeman, D. (éds), Des topoï à la théorie des stéréotypes en passant par la polyphonie et l'argumentation dans la langue. Hommages à Jean-Claude Anscombre. Université de Savoie, pp. 169180.

Michaux, Ch., (1999) « Le proverbe : nom ou phrase ? », II Congreso Internacional de Paremologia, in Parémia. N8, Cordoba, Espagne, pp. 339-344.

Petitjean, A. \& S. Petillon, (2013) « De l'usage de la parole proverbiale dans les textes dramatiques », in Pratiques. N¹59-160 (« Le Figement en débat»), pp. 295-309.

Rius-Dalmau, M.-I., (2014) «Enseigner et apprendre les unités parémiologiques d'une langue étrangère : du XIXe siècle à nos jours », in Cédille. $\mathrm{N}^{\circ} 10$, pp. 333-344.

Rodegem, F. \& P. Van Brussel, (1989) « Proverbes et pseudoproverbes. La logique des parémies », in Europhras. № 88, Strasbourg, éd. Gréciano, coll. Phraséologie contrastive, pp. 349-356.

Saussure, F. de, (1974) Cours de linguistique générale. Paris, Payot (1 ère éd. 1967).

Schapira, Ch., (2000) « Proverbe, proverbialisation et déproverbialisation », in Langages. $\mathrm{N}^{\circ} 139$, pp. 81-97.

Sułkowska, M., (2013) De la phraséologie à la phraséodidactique. Études théoriques et pratiques. Wydawnictwo Uniwersytetu OEl skiego Katowice.

Sullet-Nylander, F., (2005) « Jeux de mots et défigements à La Une de Libération (19732004) », in Langage et société. $\mathrm{N}^{\circ} 112$, pp. 111-139.

Théophanous, O., (2007) « Le proverbe comme entre-deux : aspects linguistiques et interculturels », in Collès, L. (et al.) (dir.), Didactique du FLE et de l'interculturel. Littérature, biographie langagière et médias. EME Éditions, pp. 109-121.

Villiers, D., (2010) « Les modalités du détournement proverbial : entre contraintes et libertés », in Modèles linguistiques. N62, pp. 147-172. 
Wozniak, A., (2009) « Le proverbe détourné : étude théorique appliquée à un corpus bilingue franco-espagnol », in Paremia. № 18, pp. 185-196.

\section{Articles de presse cités}

\section{Libération}

15/4/1998 : Coroller, Catherine, « Noël au balcon, choux-fleurs à foison »

12/8/1998 : Caillat, Sophie, « Tous les chemins mènent au TRO Breiz »

16/4/1999 : «Quand le vin est tiré », Ressources informatisées de la presse (Base de données

Factiva)

4/10/2000 : ARVIN-BEROD, Alain. « L'habit ne fait pas le moine... ni le républicain ».

21/6/2001 : Grenier, Jean-Yves, «L'habit ne fait pas le moi »

23/11/2001 : Guibal, Claude, « Le Caire, nombril du monde »

12/10/2001 : Briet, Sylvie, « Au pays des aveugles, la boussole est reine ».

26/4/2002 : Saberanc, Haydée ; Bertrand, Olivier ; Davidenkoff, Emmanuel et al, « Cours de Banderoles en Province »

\section{Le Figaro}

9/10/2001 : Pessis, Jacques, « Il faut battre le fer quand il est chaud, et la crème quand elle est fraîche "

8/12/2001 : Morice, Michel, «Quand le chat n'est pas là »

17/11/2004 : «Quand le vin est tiré ». Ressources informatisées de la presse (Base de données Factiva)

\section{Les Échos}

18/11/1998 : « La décentralisation en panne sèche ». Ressources informatisées de la presse (Base de données Factiva)

02/07/2004 : Chevilly, Philippe, «Souffle Tragique »[En ligne]. Disponible sur : http:// www.lesechos.fr/02/07/2004/LesEchos/19192-515-ECH_souffle-tragique.htm [Dernier accès le 3 février 2016].

\section{Le Matin}

1/12/2007 : Habimana, « Quand le chat n'est pas là ou qu'il dort sous un palmier, les souris dansent $\gg$

27/9/2008 : «Quand le chat n'est pas là ». Ressources informatisées de la presse (Base de données Factiva)

\section{Le Temps}

8/10/2008 : Creutz, Norbert, "Au pays des aveugles, le cinéma s'égare »

\section{Le Progrès}

21/11/2003 «Quand le vin est tiré ». Ressources informatisées de la presse (Base de données Factiva)

14/3/2007 : Décot, Philippe, « Quand le chat n’est pas là ».

\section{L'Équipe}

8/6/2007 : Dégoulet, Guillaume, «Quand le chat n'est pas là ». 


\section{Midi Libre}

16/11/2007 : Diez, René, « Des Africaines sur le trottoir, sous l’œil du Marabout »,

\section{Sud-Ouest}

11/12/2004 : Mansat, Dominique Couvreur, Gilles Dupuis, Emmanuel Rubin, François Simon, Sylvain Verut, Colette, "Quand le vin est tiré »

\section{Ouest-France}

« Rira bien qui rira le premier » Les Embuscades, un supplément. Ressources informatisées de la presse (Base de données Factiva)

\section{L'Yvonne républicaine-sud}

30/08/2010 : «Au pays des aveugles, il n'y a que 'l'œil du web' qui puisse être roi ». Ressources informatisées de la presse (Base de données Factiva)

\section{L'Est Républicain}

13/10/2008 : Bouhey, Cécile, « Rira bien qui rira le premier»

\section{L'Agéfi}

30/10/1996 : «Lutter contre les sociétés boîtes à lettres ». Ressources informatisées de la presse (Base de données Factiva)

\section{Automobile}

19/10/2006 : Liabaud, Pierre, « Quand le chat n'est pas là ».

\section{Le Maine libre}

24/11/2012 : «A l'UMP, à chaque jour suffit sa haine ». Ressources informatisées de la presse (Base de données Factiva)

\section{Africa Manager}

20/12/2012 : Boumiza, Khaled, «Il y en Tunisie au moins 2 millions de koffar, mécréants ». Ressources informatisées de la presse (Base de données Factiva)

\section{Reuters}

24/5/2001 : «L'habit ne fait pas la Karateka ». Ressources informatisées de la presse (Base de données Factiva)

\section{La Tribune de Genève}

16/2/2008 : « Sans le chat, les souris dépriment ! ». Ressources informatisées de la presse (Base de données Factiva)

16/2/2008 : « Rira bien qui rira le premier ». Ressources informatisées de la presse (Base de données Factiva). 